Supporting Information

\title{
Facile Post-Carboxymethylation of Cellulose Nanofiber Surfaces for Enhanced Water Dispersibility
}

\author{
Hiroyuki Kono, ${ }^{* \dagger}$ Eiki Tsukamoto, ${ }^{\dagger}$ and Kenji Tajima ${ }^{\ddagger}$ \\ 'Division of Applied Chemistry and Biochemistry, National Institute of Technology, Tomakomai \\ College, Nishikioka 443, Tomakomai, Hokkaido 059 1275, Japan \\ tFaculty of Engineering, Hokkaido University, N13W8, Kita-ku, Sapporo, Hokkaido 0608628 , \\ Japan
}



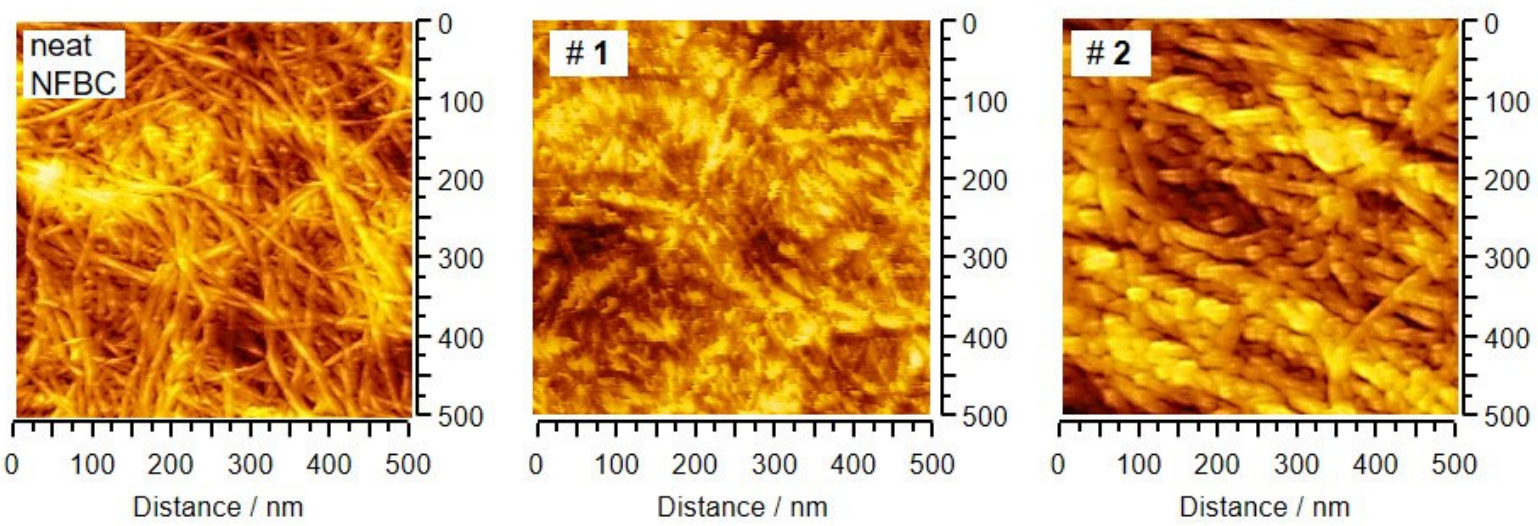

Figure S1. SPM images of neat NFBC and CMNFBC \#1 and \#2.

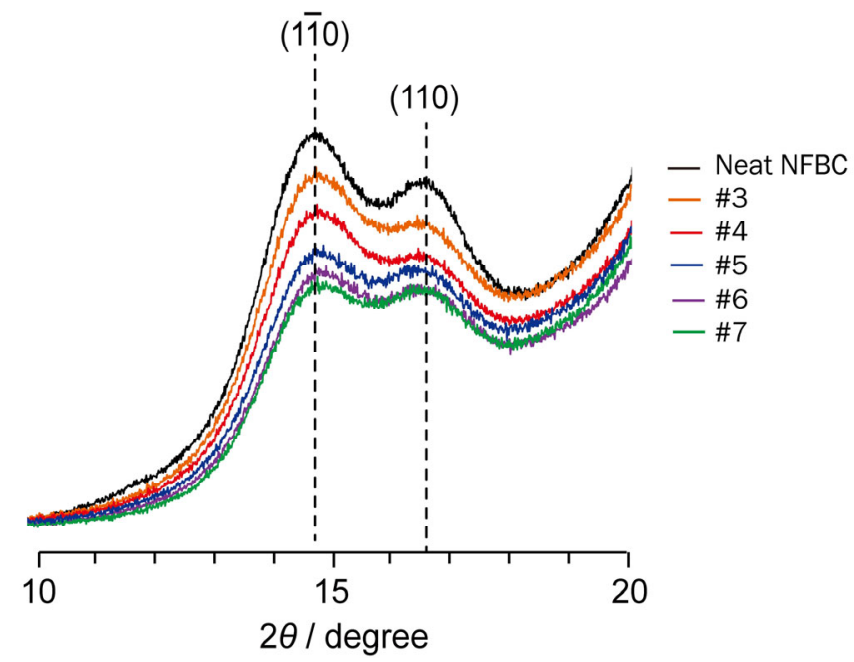

Figure S2. Intensity of (11-0) crystal plane of cellulose I in the XRD patterns of CMNFBC \#3-7 and neat NFBC. The peak heights of the (11-0) plane were normalized by that of (200) plane at 22. $2^{\circ}$ (Figure 5). 

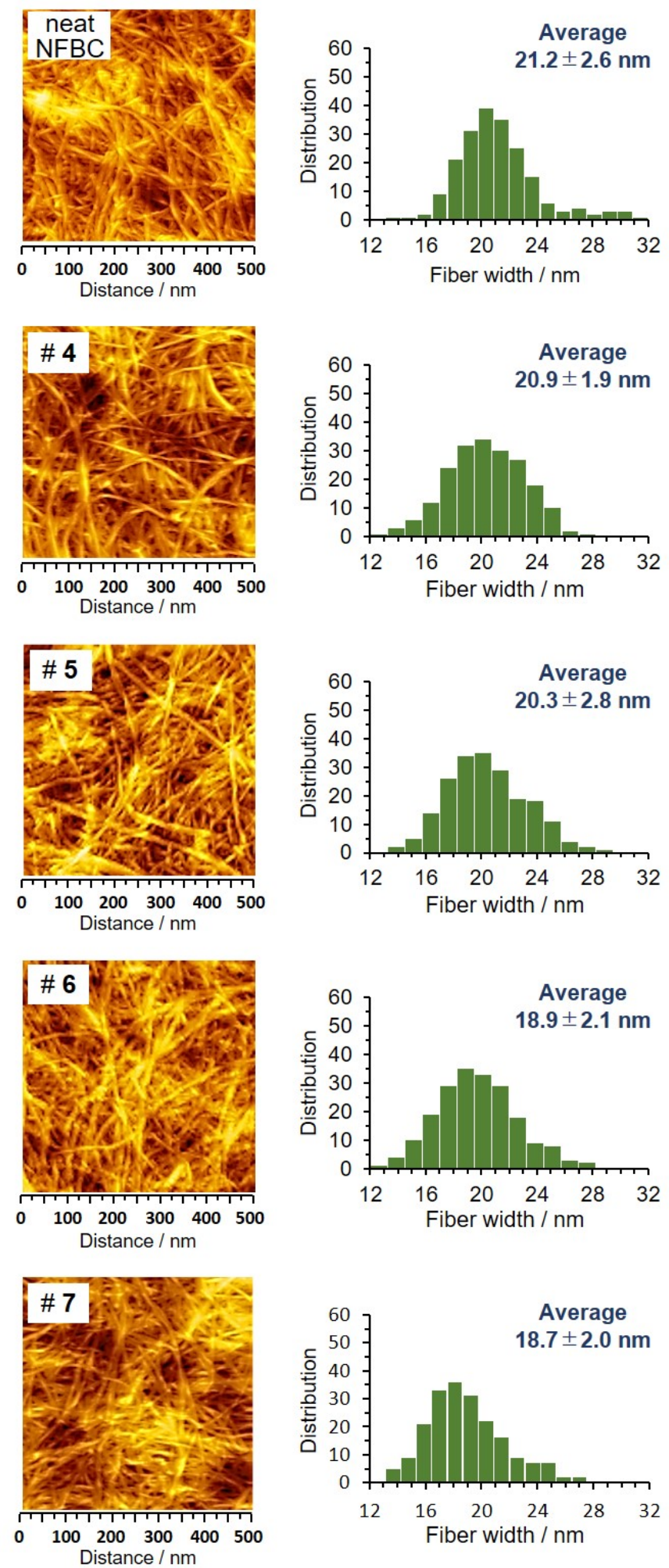

Figure S3. SPM images and fiber width distributions $(n=100)$ of neat NFBC and CMNFBC \#47. 


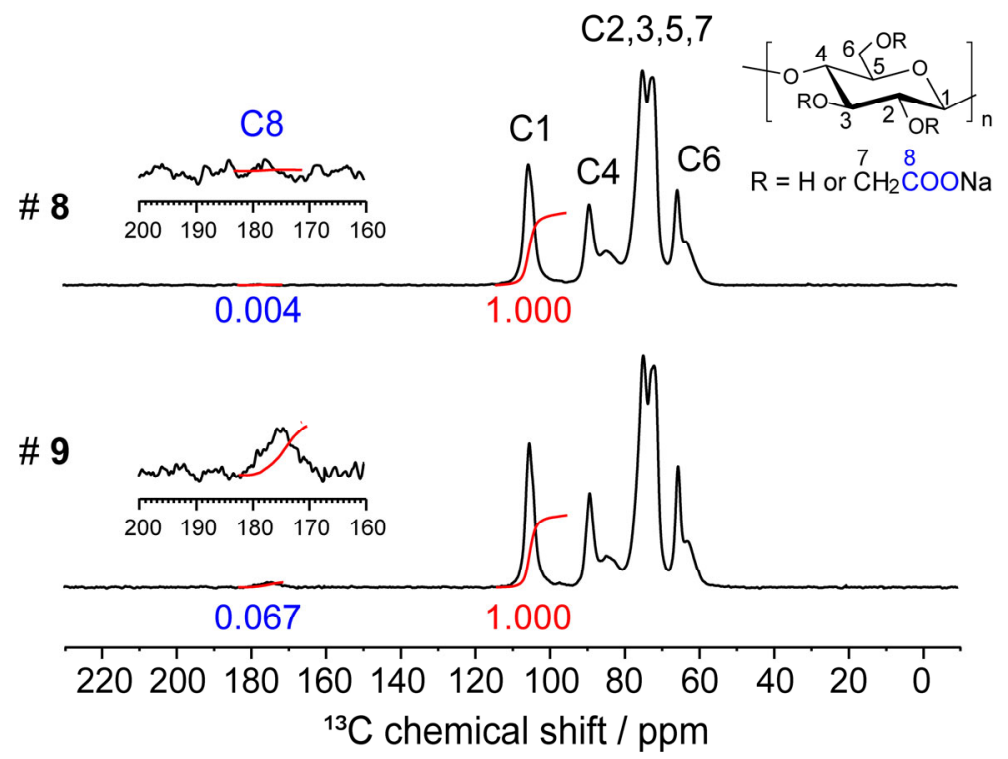

Figure S4. Solid-state ${ }^{13} \mathrm{C}$ NMR spectra of CMNFBC \#8 and \#9. The DS was determined from the integral value of the carbonyl carbon $(\mathrm{C} 8)$ resonance relative to that of the $\mathrm{C} 1$ resonance, which was set to 1.

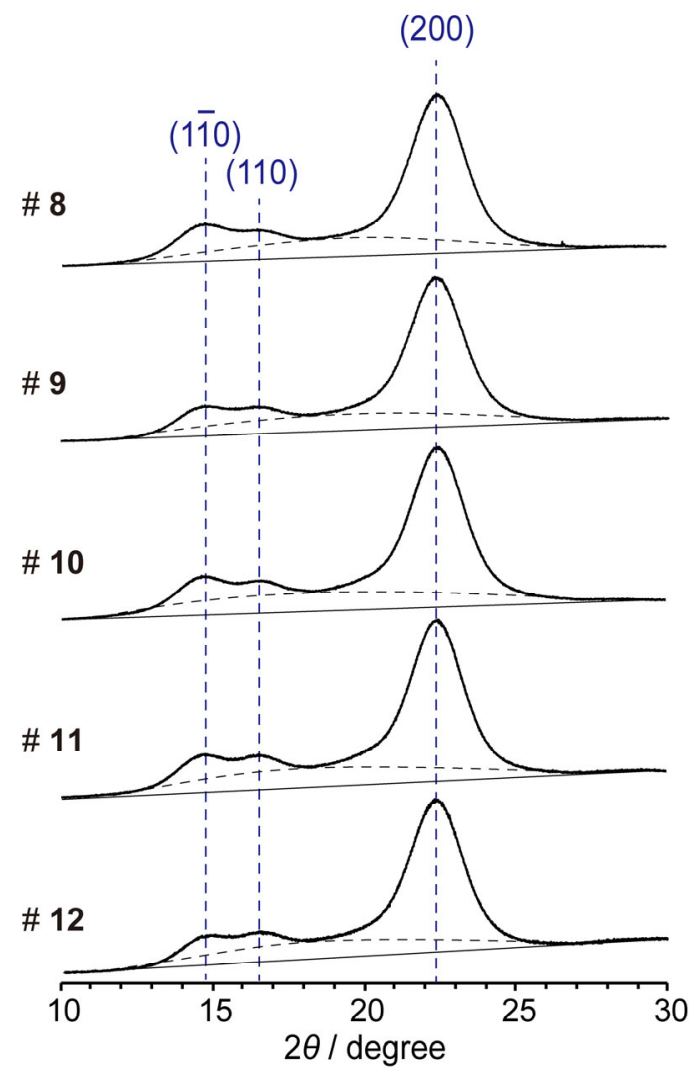

Figure S5. XRD patterns of CMNFBC \#8-12. The crystal planes for cellulose I are also indicated. 